\title{
El sistema de transporte ferroviario en el Perú: 2004-2016
}

\section{The rail transport system in Perú: 2004-2016}

\author{
Ronald Michael Mendoza Colque, Lucio Leo Verástegui Huanca ${ }^{2}$
}

\section{RESUMEN}

Este trabajo de investigación explica y analiza de manera detallada el sistema de transporte ferroviario en los años 2004 y 2016, bajo la perspectiva de una economía de mercado competitivo. La inversión en redes de infraestructura de carga y pasajero es de vital importancia para la competitividad de un país, con ello se logra economías de escala, reduciendo los costos de transporte, y un incremento en el dinamismo de la economía. El sistema de transporte ferroviario, es el más eficiente, menos costoso y menos contaminante, en comparación a los demás modos de transporte; por el cual, teniendo en cuenta estas ventajas, la inversión en este sector podría desencadenar un impacto mayor en la economía y en el bienestar social.

Palabras clave: transporte ferroviario, economías de red, cuota de mercado, mercado competitivo.

\begin{abstract}
This working paper explains and analyzes in detail the railway transport system between 2004 and 2016; focusing on the perspective of a competitive market economy. Investment in cargo and passenger infrastructure networks is vital for a country's competitiveness, thereby achieving economies of scale, reducing transportation costs, and an increase in the dynamism of the economy. The rail transport system is the most efficient, least expensive and least polluting, compared to other kinds of transportation; by this, paying attention to these advantages, investment in this sector could trigger a greater impact on the economy and social welfare.
\end{abstract}

Keywords: rail transport, network economies, market share, competitive market.

\footnotetext{
${ }^{1}$ Magister en Economía, Regulación y Competencia en los Servicios Públicos en la Universitat de Barcelona. Correo electrónico: rmendoza1286@ gmail.com

${ }^{2}$ Magister en Economía Internacional y Desarrollo Económico en la Universidad Complutense de Madrid. Correo electrónico:iverasteguicorp@gmail.com
} 


\section{INTRODUCCIÓN}

El nivel de competitividad de un país está estrechamente relacionado con el nivel de inversión y desarrollo de la infraestructura. La modernización de la infraestructura de medios de transporte, como los ferrocarriles permite interconectar los centros de producción, los centros de distribución y el consumo con mayor eficacia y menores costos de operación (CEPAL, 2012).

En el sector de transportes la forma predominante de privatización, ha sido la transferencia de la responsabilidad por los servicios y la inversión en infraestructura a instituciones privadas, sobre la base de concesiones a largo plazo. Un prerrequisito de las concesiones ferroviarias es la separación no solamente entre la infraestructura y las operaciones, sino también entre los dos tipos de servicio - de pasajeros y de carga - de forma que sea posible otorgar concesiones por separado. En casos importantes los gobiernos han levantado una gran parte de las regulaciones $\mathrm{y}$, al mismo tiempo, han transferido al sector privado la administración, tanto de obras de infraestructura como de servicios de transporte, que anteriormente habían sido ofrecidos por el sector estatal y que intrínsecamente, por distintas razones, no siempre se suministran en un ambiente completamente competitivo (Thomson, 2001).

Los ferrocarriles son un medio de transporte eficiente, que concentra la gente y las mercancías y los transporta en una ruta fija, utilizando una máquina locomotora y múltiples coches y vagones de carga. El transporte es generalmente más económico en combustible que el transporte carretero (Banco Mundial, 2011; Ruiz-Rua, 2010). Los transportes ferroviarios han sido con frecuencia considerados como un servicio público al igual que el suministro de agua, de gas o de electricidad, lo que ha causado resultados indeseables de políticas y de gestión (Banco Mundial, 2011).

En 1999, las vías férreas en el Perú estaban compuestas principalmente por el Ferrocarril Central, el del Sur, y el del Sur Oriente. Estas líneas estaban administradas por la Empresa Nacional de Ferrocarriles (ENAFER) de propiedad del Estado. Luego se implementó la modalidad de consorcios divisibles que consistía en juntar todos los sistemas ferroviarios en el proceso de subasta para un consorcio de empresas que una vez que recibiera la adjudicación se separaría en las distintas empresas que lo componen. Se otorgó un contrato de concesión de treinta años prorrogables hasta sesenta años al Consorcio de Ferrocarriles del Perú, que se dividió en dos operadores: Ferrovías Central Andina S.A. para operar el ferrocarril central y Ferrocarril Transandino S.A. para operar los otros dos ferrocarriles.

Entre tanto, este estudio tiene como objetivo de analizar, y explicar el sistema de transporte ferroviario en el Perú entre los años 2004 y 2016, desde una perspectiva de una economía de mercado; partiendo del supuesto, que después del proceso de la liberalización, el sistema del transporte ferroviario ha evolucionado favorablemente hasta acercarse a un mercado competitivo, y que el dinamismo en este mercado ha tenido una tendencia creciente, tanto en inversiones, traslado de carga y pasajeros, con un bienestar social de mercado mucho mayor.

\section{MATERIAL Y MÉTODOS}

Para garantizar el funcionamiento eficiente de los mercados, frecuentemente es necesaria la aplicación de una regulación económica para corregir ciertos fallos de mercado o incentivarles a realizar alguna acción o modificar las decisiones de millones de personas o de un grupo de empresas, para que estos efectos sean congruentes con los objetivos del gobierno. Por otra parte, los gobiernos no siempre se han mostrado suficientemente cuidadosos o valientes en identificar y enfrentar las tendencias anticompetitivas que pueden existir en los mercados. En el fondo, esto no constituye una deficiencia de los mercados sino una falla en su regulación (Thomson, 2001).

En el año 1998 se crea el Organismo Supervisor de la Inversión de la Infraestructura de Transporte de Uso Público(OSITRAN), como organismo público con la finalidad de regular el comportamiento de los mercados en los que actúan las entidades prestadoras, así como, el cumplimiento de los contratos de concesión, cautelando en forma imparcial y objetiva los intereses del estado, de los inversionistas y de los usuarios en el marco de las políticas y normas que dicta el Ministerio de Transportes y Comunicaciones (MTC), a fin de garantizar la eficiencia en la explotación de la infraestructura bajo su ámbito.

A diferencia de otros contratos de concesión de infraestructura de transporte supervisados por OSITRAN, los contratos con Ferrovías Central Andina y Ferrocarril Transandino S.A. (FETRANSA) no contemplan la realización de inversiones obligatorias en la infraestructura ferroviaria o en las zonas de influencia de la concesión, lo cual impide la identificación de metas de inversión específicas. Sin embargo, dado que ambos contratos exigen alcanzar los requisitos y estándares de la United States Federal Railroad 
Administration (FRA) Class II, a más tardar dentro de los cinco primeros años de la concesión, se han considerado como necesidades de inversión aquellas inversiones que se requieran para alcanzar esta meta (Pastor \& Pérez, 2009).

Según el estudio realizado por Thomson (2001) un mercado reglamentado mediante concesiones de operación de servicios podría mostrar señales de anti competitividad, si la duración de la concesión es larga y no hay posibilidades de ingreso de nuevos operadores al servicio. Hay un inadecuado monitoreo de los mercados de servicios de transporte para determinar hasta qué punto están dominados por una sola empresa, o grupo de empresas y, evidentemente, se han tomado pocas medidas para evitar que sucedan acciones anticompetitivas, aún en países donde existen leyes antimonopolio.

Entonces el problema que surge es cómo regularlo de manera que garantice la competitividad del mercado y beneficios sociales que superen los costos sociales causados, todo ello sin estrangular la mayor eficiencia que la privatización y la desreglamentación están destinadas a generar y permitir que las empresas privadas usen toda su capacidad de iniciativa para desarrollar mercados y reducir costos (Thomson, 1998).

Una reforma de los ferrocarriles significa todo cambio significativo en la política del gobierno, en la estrategia de inversiones o en la estructura de gestión, que busque mejorar el rendimiento del sistema ferroviario. Los ferrocarriles son instituciones complejas que poseen múltiples herramientas de medición del rendimiento - costos, tarifas de transporte, niveles de servicio y necesidades de inversión, entre otros factores (Banco Mundial, 2011).

Históricamente, la mayoría de los países escogieron políticas que restringían la competencia entre las compañías de ferrocarril. Actualmente, cada vez más países eligen políticas que permiten la competencia en el sector de carga ferroviario y, más raramente, en el sector de los servicios de pasajeros inter-ciudades (Banco Mundial, 2011). La regulación y la reforma estructural implica una regulación de acuerdo al contexto y al sector que se va a aplicar (Gwilliam, 2001).

En los Estados Unidos, el problema se resuelve, esencialmente, definiendo una tasa de rentabilidad razonable para la empresa monopolista. En el Reino Unido, el principio preferido es el de fijar la escala de tarifas que la empresa está autorizada a cobrar por un período de cinco años, expresada en un número de puntos porcentuales inferior o superior al índice de precios al consumidor. Las dos opciones tienen sus ventajas y desventajas. La estadounidense permite asegurar el interés de los inversionistas y mantener la rentabilidad aunque haya fluctuaciones inesperadas en el costo de los insumos, pero si la rentabilidad permitida se fija en un valor un poco superior al de mercado, podría incentivar a las empresas a hacer inversiones innecesarias, meramente para maximizar la rentabilidad de su capital, Bajo la opción británica, las empresas tendrían mayores incentivos para reducir sus costos, pero a veces no es fácil definir los reajustes autorizados en sus tarifas (Thomson, 1998).

En Alemania se ha tenido dos categorías bien definidas. Primero, todas las categorías de regulación que son básicas para que el mecanismo del mercado y el crecimiento de la economía vayan de la mano, como por ejemplo derechos de propiedad privada, leyes de comercio, leyes anti cartel, leyes civiles y criminales, certificados de título, seguridad, seguridad social, y el cuidado del medio ambiente. Estas regulaciones reducirán los costos de transacción. Segundo, la otra categoría tiene que ver con la regulación en industrias específicas, compañías, o mercados. Los instrumentos regulatorios que típicamente son empleados son restricciones de entrada y salida al mercado, precios, producción e inversión, términos de contrato (Donges, 1991).

Entre los años 1850 y 1964 se construyeron en el Perú, 42 líneas férreas, que alcanzaron un total de $4.257 \mathrm{~km}$. La influencia de diferentes factores ha sido la causa que este sector se descomponga, y disminuya el servicio de transporte para el año 2014, teniendo la red solo tenía una extensión de $1.939,7 \mathrm{~km}$. Con esto, el Perú es uno de los países con menor desarrollo ferroviario de la región. Lamentablemente, este medio de transporte no ha formado parte de las políticas viales de los gobiernos anteriores y no ha sido considerado como una alternativa viable al transporte por carreteras (Pastor \& Pérez, 2009). El análisis de la evidencia empírica muestra una relación inversa entre el crecimiento de un país y sus costos de transporte. Así, países con elevados costos de transporte tienden a crecer menos que aquellos con bajo costo de transporte (Ruiz \& Ludeña, 2008).

El sistema ferroviario peruano es un sistema no integrado con el resto de los modos de transporte y se caracteriza por su muy baja densidad. El promedio latinoamericano es de $43 \mathrm{Km}$ de rieles cada 1.000 $\mathrm{Km} 2$ de superficie, mientras que en Perú esta relación sólo alcanza 13,9 (CAF, 2004).

Los transportes eficientes son un componente crítico del desarrollo económico, al nivel nacional tanto como mundial. La disponibilidad de transportes 
afecta los patrones de desarrollo mundial, y puede fomentar o poner obstáculos al crecimiento económico dentro de cada nación individualmente (Banco Mundial, 2011). Pero no es solamente inversión en infraestructura física lo que se requiere para mejorar las condiciones de transporte en América Latina, sino, también, mejorar las condiciones de operación, de forma que la regulación del transporte, la libre competencia y la regulación formen un círculo virtuoso que permita satisfacer las necesidades individuales, generando rentabilidades razonables a los operadores del transporte (Rufián, 2002).

Debido a que la mayoría de los operadores del transporte son inversionistas privados, el rol del sector público es crear las condiciones para que el sector privado pueda ofrecer servicios de calidad, en un entorno competitivo (Rufián, 2002).

En el año 2008 la longitud total del sistema de red ferroviaria llegó a $1.927 \mathrm{Km}$ y en el año 2010 llegaba solo a una extensión de 1.906,6 km. En el año 1999 fue concesionada al Consorcio de Ferrocarriles del Perú, que se dividió en dos operadores: Ferrovías Central Andina S.A. para operar el Ferrocarril Central y Ferrocarril Transandino S.A. (FETRANSA) para operar la ruta sur (Matarani-Juliaca, Juliaca-Cusco y Juliaca-Puno), cuya principal actividad es el transporte de carga, así como la ruta sur-oriente (Cusco-Macchu Picchu), cuyo principal rubro es el transporte de pasajeros (Pastor \& Pérez, 2009).

El transporte ferroviario ofrece grandes ventajas como la gran capacidad de carga por eje, la menor tasa de accidentabilidad, el ahorro de combustible, la menor cantidad de emisiones contaminantes y la descongestión de las carreteras. Además constituye el transporte masivo más eficiente de los últimos años.

Tabla 1. Principales vías férreas concesionadas por OSITRAN.

Infraestructura

Entidad prestadora

Plazo

Modalidad

\begin{tabular}{llcr}
\hline Ferrocarril del Centro & Ferrovías Central Andina & 40 años & Autosostenible \\
\hline Ferrocarril del Sur y Sur Oriente & $\begin{array}{l}\text { Consorcio Ferrocarril Trasandino } \\
\text { S.A. }\end{array}$ & 35 años & Autosostenible \\
& & & \\
\hline Metro de Lima - Línea 1 & GYM Ferrovías S.A. & 30 años & Cofinanciada \\
\hline $\begin{array}{l}\text { Metro de Lima y Callao - Línea } \\
2\end{array}$ & Consorcio Nuevo Metro de Lima & 35 años & Cofinanciada \\
& S.A. & &
\end{tabular}

Fuente: MTC-DGCF-MTC-OGPP-Oficina de Estadística: 2010-2016.

La variación de la longitud de las líneas ferroviarias o la inversión en nuevas rutas no ha sido relevante. Desde el año 2010 hasta el año 2016, la variación total de la longitud de las líneas ferroviarias solo ha sido de $1.74 \%$, lo cual no tiene un impacto relevante en la integración de ciudades a través de la red vial ferroviaria. En términos absolutos solo existe una variación de $33.1 \mathrm{~km}$; lo que específicamente representa la construcción del Metro de Lima-Línea 1. Las demás líneas - el ferrocarril del centro, ferrocarril del Sur y Sur Oriente - no tienen ninguna variación en su extensión. Tal como se puede ver en la tabla 2. 
Tabla 2. Infraestructura ferroviaria por empresa, tramo y longitud según régimen de propiedad: 2010-2016.

\begin{tabular}{|c|c|c|c|c|c|c|c|c|}
\hline \multirow{2}{*}{$\begin{array}{l}\text { Régimen de } \\
\text { propiedad }\end{array}$} & \multirow[t]{2}{*}{ Empresa y tramo } & \multicolumn{7}{|c|}{ Longitud (Km) } \\
\hline & & 2010 & 2011 & 2012 & 2013 & 2014 & 2015 & 2016 \\
\hline Total & & 1906,6 & 1927,5 & 1927,5 & 1927,5 & 1939,7 & 1939,7 & 1939,7 \\
\hline \multirow{4}{*}{$\begin{array}{l}\text { Público no } \\
\text { concesionado }\end{array}$} & Gobierno Regional de Tacna & 60,0 & 60,0 & 60,0 & 60,0 & 60,0 & 60,0 & 60,0 \\
\hline & Tacna - Arica & 60,0 & 60,0 & 60,0 & 60,0 & 60,0 & 60,0 & 60,0 \\
\hline & $\begin{array}{l}\text { Ministerio de Transportes y } \\
\text { Comunicaciones }\end{array}$ & 128,7 & 128,7 & 128,7 & 128,7 & 128,7 & 128,7 & 128,7 \\
\hline & Huancayo - Huancavelica & 128,7 & 128,7 & 128,7 & 128,7 & 128,7 & 128,7 & 128,7 \\
\hline \multirow{16}{*}{$\begin{array}{c}\text { Público } \\
\text { concesionado }\end{array}$} & Ferrovías Central Andina & 489,6 & 489,6 & 489,6 & 489,6 & 489,6 & 489,6 & 489,6 \\
\hline & Callao - La Oroya & 222,0 & 222,0 & 222,0 & 222,0 & 222,0 & 222,0 & 222,0 \\
\hline & La Oroya - Huancayo & 124,0 & 124,0 & 124,0 & 124,0 & 124,0 & 124,0 & 124,0 \\
\hline & La Oroya - Cerro de pasco & 132,0 & 132,0 & 132,0 & 132,0 & 132,0 & 132,0 & 132,0 \\
\hline & $\begin{array}{l}\text { Cut off (Callao-La Oroya)- } \\
\text { Huascacocha }\end{array}$ & 11,6 & 11,6 & 11,6 & 11,6 & 11,6 & 11,6 & 11,6 \\
\hline & Ferrocarril Transandino & 989,7 & 989,7 & 989,7 & 989,7 & 989,7 & 989,7 & 989,7 \\
\hline & Matarani - Arequipa & 147,5 & 147,5 & 147,5 & 147,5 & 147,5 & 147,5 & 147,5 \\
\hline & Arequipa - Juliaca & 304,0 & 304,0 & 304,0 & 304,0 & 304,0 & 304,0 & 304,0 \\
\hline & Juliaca - Puno & 47,7 & 47,7 & 47,7 & 47,7 & 47,7 & 47,7 & 47,7 \\
\hline & Juliaca - Cusco & 337,9 & 337,9 & 337,9 & 337,9 & 337,9 & 337,9 & 337,9 \\
\hline & Empalme - Mollendo & 17,9 & 17,9 & 17,9 & 17,9 & 17,9 & 17,9 & 17,9 \\
\hline & $\begin{array}{l}\text { Cusco - Hidroeléctrica } \\
\text { Machupicchu }\end{array}$ & 121,7 & 121,7 & 121,7 & 121,7 & 121,7 & 121,7 & 121,7 \\
\hline & Pachar - Urubamba & 13,0 & 13,0 & 13,0 & 13,0 & 13,0 & 13,0 & 13,0 \\
\hline & GYM Ferrovías & 0,0 & 20,9 & 20,9 & 20,9 & 33,1 & 33,1 & 33,1 \\
\hline & $\begin{array}{l}\text { Villa El Salvador - Estación } \\
\text { Grau }\end{array}$ & 0,0 & 20,9 & 20,9 & 20,9 & 20,9 & 20,9 & 20,9 \\
\hline & $\begin{array}{l}\text { Estación Grau - Estación } \\
\text { Bayovar }\end{array}$ & 0,0 & 0,0 & 0,0 & 0,0 & 12,2 & 12,2 & 12,2 \\
\hline \multirow{7}{*}{ Privado } & Cemento Andino & 13,6 & 13,6 & 13,6 & 13,6 & 13,6 & 13,6 & 13,6 \\
\hline & Caripa - Condorcoha & 13,6 & 13,6 & 13,6 & 13,6 & 13,6 & 13,6 & 13,6 \\
\hline & $\begin{array}{l}\text { Southern Perú Copper } \\
\text { Corporation }\end{array}$ & 217,7 & 217,7 & 217,7 & 217,7 & 217,7 & 217,7 & 217,7 \\
\hline & Ilo - Toquepala & 186,0 & 186,0 & 186,0 & 186,0 & 186,0 & 186,0 & 186,0 \\
\hline & El Sargento - Cuajone & 31,7 & 31,7 & 31,7 & 31,7 & 31,7 & 31,7 & 31,7 \\
\hline & Votorantim Metais & 7,3 & 7,3 & 7,3 & 7,3 & 7,3 & 7,3 & 7,3 \\
\hline & Santa Clara - Cajamarquilla & 7,3 & 7,3 & 7,3 & 7,3 & 7,3 & 7,3 & 7,3 \\
\hline
\end{tabular}

Fuente: MTC-DGCF-MTC-OGPP-Oficina de Estadística: 2010-2016. 
Las economías de red tienen la característica de tener un componente esencial que va a ser difícil de replicar por un nuevo competidor que quisiera entrar al mercado. El cual no solamente restringiría la competencia, sino que también, la opción pueda ser que solo operando en el mercado una empresa puede ser la óptima. El dominio de mercado aparece cuando la empresa existente en el mercado tiene un poder de mercado y en algunas situaciones puede imponer precios muy cercanos a los de monopolio.

Un potencial para incrementar la competencia en industrias sujetos a la regulación son los mercados contestables (Baumol, Panzar and Willig 1982). Si la entrada al mercado es libre, el involucrado no podrá tener un monopolio en la inversión, producción, y decisión en el precio, porque sería excluido del mercado por la entrada del nuevo competidor. El rápido cambio tecnológico, especialmente la adquisición de información nueva y los medios de comunicación, incrementa tener un mercado altamente contestable. Al mismo tiempo, la importancia de los costos hundidos de entrada y las barreras de salida serian irrelevantes (Donges, 1991).

En el caso del transporte terrestre de carga en el Perú se emplean tanto las carreteras como los ferrocarriles, sin embargo, la mayor parte de la carga se moviliza por la red vial, a pesar de que el tren es más eficiente que la red vial para movilizar grandes volúmenes de carga en largas distancias (Pastor \& Pérez, 2009).

Un ferrocarril moderno cuenta con una gran capacidad de carga que equivale a diez carreteras de doble vía aproximadamente. Respecto al costo de construcción y operación, el costo de la vía ferroviaria y sus obras se estima en un tercio del costo de una carretera, siendo además que un ferrocarril estándar consume menos energía que la correspondiente a un vehículo en carretera que movilice la misma carga (Pastor \& Pérez, 2009).

Los ferrocarriles utilizan una tecnología única con un coeficiente de fricción muy bajo, y basada en rieles y ruedas de acero. Las fuerzas más difíciles de superar, a parte del peso de las mercancías transportadas, son la resistencia al rodamiento y la resistencia aerodinámica a medida que la velocidad aumenta. Después del transporte fluvial, el transporte ferroviario es el medio más eficaz para mover grandes volúmenes de mercancías o de pasajeros (Banco Mundial, 2011).

Las ventajas de costo de un transporte ferroviario bien administrado pueden fomentar la competitividad en los mercados nacionales y mundiales de los fabricantes y de los expedidores, y ejercer una presión competitiva en los precios del transporte vial. Asimismo, un transporte de pasajeros eficiente y bien administrado puede aumentar la movilidad de la mano de obra, ampliando la base de mano de obra y el desarrollo desde los centros urbanos hacia el exterior(Banco Mundial, 2011).

El problema económico del sistema de transporte ferroviario aparece aparentemente cuando compite con el sistema de transporte vial, y que este debe generar excedentes para cubrir los costos de operación (Thomson, 1999).

\section{RESULTADOS}

La participación del sistema de transporte ferroviario, tanto en carga como en pasajeros, es variada. Para el año 2004, un total de 1.351.924 personas han utilizado el servicio ferroviario para desplazarse de un lugar a otro; mientras que esta cifra para el año 2016, ha incrementado a 2.773.234, el cual constituye un crecimiento de $105.13 \%$, entre los años 2004 y 2016. En el servicio de pasajeros, la concentración del servicio lo tiene el operador Perú Rail, teniendo una cuota de mercado bastante alta, en los 2004 y 2016 . Tal como se ve en la figura 1.

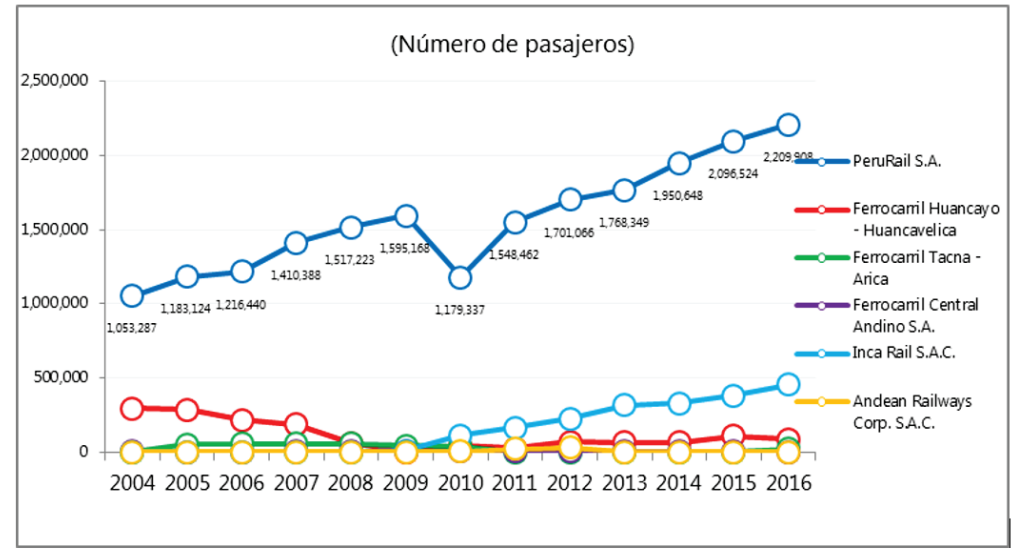

Figura 1. Tráfico Ferroviario de Pasajeros, según operador: 2004-2016 
Fuente: Empresas Ferroviarias. Elaboración: MTC - OGPP - Oficina de Estadística: 2004-2016.

El operador Perú Rail S.A. concentra en promedio, un $84 \%$ de la totalidad del servicio de traslado de pasajeros entre los años 2004 y 2016. Perú Rail S.A., presta los servicios en los tramos de Matarani - Cusco y Cusco Hidroeléctrica, dentro de estos dos tramos, la mayor concentración en el traslado de pasajeros se acentúa, en el tramo Cusco - Hidroeléctrica, teniendo un promedio del 98\%, por ser zona turística. El operador Inka Rail S.A.C. y el ferrocarril Huancayo-Huancavelica, tienen una participación del 7\% del traslado de pasajeros, y el resto lo concentra las demás operadoras. La brecha es bastante diferenciada, y bastante segmentada geográficamente. Tal como se puede ver en la tabla 3.

Tabla 3. Tráfico ferroviario de pasajeros, según operador, 2004-2016 (\% número de pasajeros)

\begin{tabular}{|c|c|c|c|c|c|c|c|c|c|c|c|c|c|}
\hline Operador & 2004 & 2005 & 2006 & 2007 & 2008 & 2009 & 2010 & 2011 & 2012 & 2013 & 2014 & 2015 & 2016 \\
\hline Total & $100 \%$ & $100 \%$ & $100 \%$ & $100 \%$ & $100 \%$ & $100 \%$ & $100 \%$ & $100 \%$ & $100 \%$ & $100 \%$ & $100 \%$ & $100 \%$ & $100 \%$ \\
\hline Perú Rail S.A. & $78 \%$ & $78 \%$ & $82 \%$ & $85 \%$ & $93 \%$ & $97 \%$ & $85 \%$ & $88 \%$ & $84 \%$ & $82 \%$ & $83 \%$ & $81 \%$ & $80 \%$ \\
\hline Matarani - Cusco & $3 \%$ & $3 \%$ & $3 \%$ & $2 \%$ & $2 \%$ & $1 \%$ & $1 \%$ & $1 \%$ & $1 \%$ & $1 \%$ & $1 \%$ & $1 \%$ & $1 \%$ \\
\hline Cusco - Hidroeléctrica & $97 \%$ & $97 \%$ & $97 \%$ & $98 \%$ & $98 \%$ & $99 \%$ & $99 \%$ & $99 \%$ & $99 \%$ & $99 \%$ & $99 \%$ & $99 \%$ & $99 \%$ \\
\hline $\begin{array}{l}\text { Ferrocarril Huancayo - } \\
\text { Huancavelica. }\end{array}$ & $22 \%$ & $19 \%$ & $15 \%$ & $11 \%$ & $4 \%$ & $0 \%$ & $3 \%$ & $1 \%$ & $3 \%$ & $3 \%$ & $3 \%$ & $4 \%$ & $3 \%$ \\
\hline Ferrocarril Tacna - Arica. & $0 \%$ & $3 \%$ & $4 \%$ & $3 \%$ & $4 \%$ & $3 \%$ & $3 \%$ & $0 \%$ & $0 \%$ & $0 \%$ & $0 \%$ & $0 \%$ & $1 \%$ \\
\hline $\begin{array}{l}\text { Ferrocarril Central } \\
\text { Andino S.A. }\end{array}$ & $0 \%$ & $0 \%$ & $0 \%$ & $0 \%$ & $0 \%$ & $0 \%$ & $0 \%$ & $0 \%$ & $0 \%$ & $0 \%$ & $0 \%$ & $0 \%$ & $0 \%$ \\
\hline Inca Rail S.A.C. & $0 \%$ & $0 \%$ & $0 \%$ & $0 \%$ & $0 \%$ & $1 \%$ & $8 \%$ & $9 \%$ & $11 \%$ & $15 \%$ & $14 \%$ & $15 \%$ & $16 \%$ \\
\hline $\begin{array}{l}\text { Andean Railways Corp. } \\
\text { S.A.C. }\end{array}$ & $0 \%$ & $0 \%$ & $0 \%$ & $0 \%$ & $0 \%$ & $0 \%$ & $0 \%$ & $1 \%$ & $1 \%$ & $0 \%$ & $0 \%$ & $0 \%$ & $0 \%$ \\
\hline
\end{tabular}

Fuente: Empresas Ferroviarias. Elaboración: MTC - OGPP - Oficina de Estadística: 2004-2016.

Con respecto al servicio de carga, en el Ferrocarril del Centro de transporta concentrados de mineral y combustibles, y, en el Ferrocarril del Sur, fundamentalmente, combustibles y granos (CAF, 2004). Para el año 2004, el tráfico ferroviario con respecto al transporte de carga fue de 7.242.549 TM; mientras que este número para el año 2016, sufriría leves cambios ascendiendo a 10.294.580 TM, teniendo una variación relativa 42.14\% y en términos absolutos, se tiene una variación de 3.052.031 TM. La concentración del tráfico ferroviario de carga está en el operador Southern Peru Copper Corporation. Tal como se ve en la figura 2.

Figura 2. Tráfico Ferroviario de Carga, según operador: 2004-2016

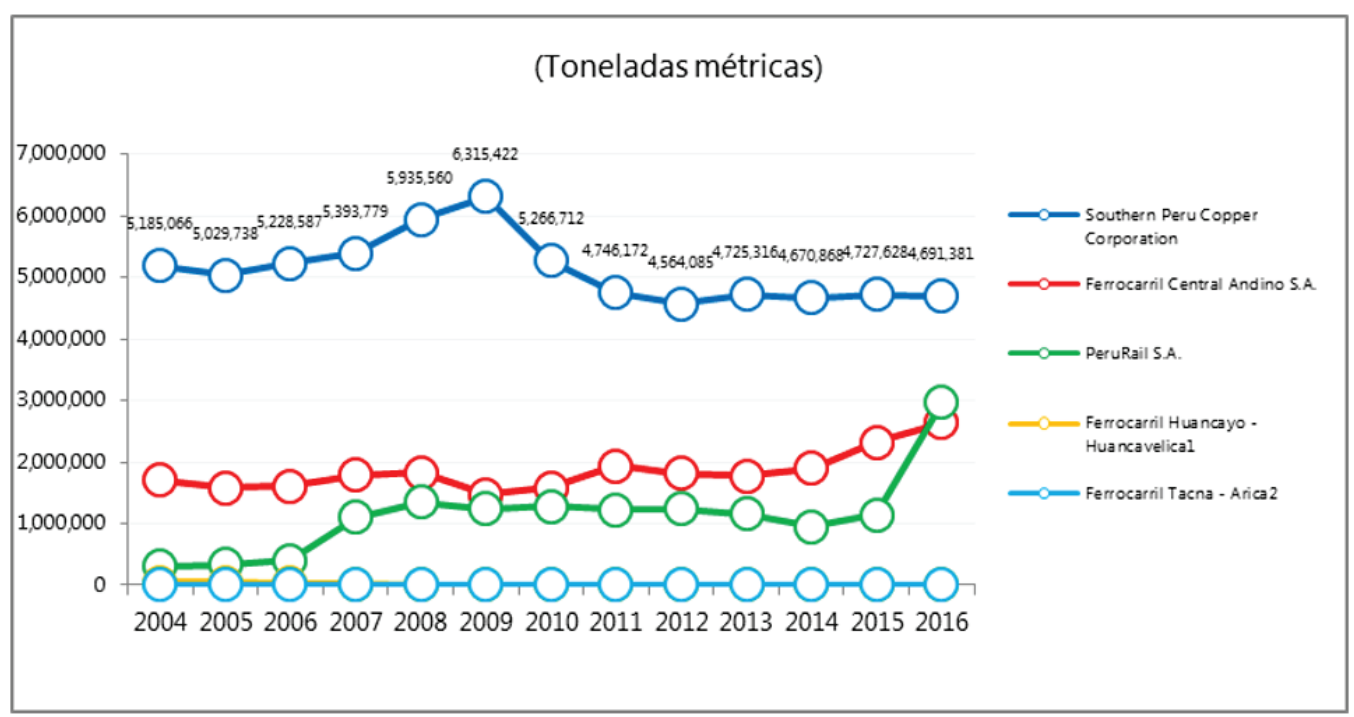

Fuente: Elaboración: MTC - OGPP - Oficina de Estadística: 2004-2016. 
La concentración o la participación en el mercado con respecto al servicio de traslado de carga, tenemos cifras bien diferenciadas. El operador Southern Peru Copper Corporation concentra en promedio un $64 \%$ de la totalidad de carga transportada en los años 2004 y 2016; mientras que el Ferrocarril Central Andino S.A. tiene una participación de $23 \%$ de la totalidad de la carga transportada, y el operador Peru Rail S.A., tiene una participación del $13 \%$. Tal como se ve en la tabla 4.

Tabla 4. Tráfico ferroviario de carga, según operador: 2004-2016 (\% toneladas métricas).

\begin{tabular}{|c|c|c|c|c|c|c|c|c|c|c|c|c|c|}
\hline Operador & 2004 & 2005 & 2006 & 2007 & 2008 & 2009 & 2010 & 2011 & 2012 & 2013 & 2014 & 2015 & 2016 \\
\hline Total & $100 \%$ & $100 \%$ & $100 \%$ & $100 \%$ & $100 \%$ & $100 \%$ & $100 \%$ & $100 \%$ & $100 \%$ & $100 \%$ & $100 \%$ & $100 \%$ & $100 \%$ \\
\hline $\begin{array}{l}\text { Southern Peru Copper } \\
\text { Corporation }\end{array}$ & $72 \%$ & $72 \%$ & $72 \%$ & $65 \%$ & $65 \%$ & $70 \%$ & $65 \%$ & $60 \%$ & $60 \%$ & $62 \%$ & $62 \%$ & $58 \%$ & $46 \%$ \\
\hline $\begin{array}{l}\text { Ferrocarril Central } \\
\text { Andino S.A. }\end{array}$ & $24 \%$ & $23 \%$ & $22 \%$ & $22 \%$ & $20 \%$ & $16 \%$ & $20 \%$ & $25 \%$ & $24 \%$ & $23 \%$ & $25 \%$ & $28 \%$ & $26 \%$ \\
\hline PeruRail S.A. & $4 \%$ & $5 \%$ & $6 \%$ & $13 \%$ & $15 \%$ & $14 \%$ & $16 \%$ & $15 \%$ & $16 \%$ & $15 \%$ & $13 \%$ & $14 \%$ & $29 \%$ \\
\hline $\begin{array}{l}\text { Ferrocarril Huancayo - } \\
\text { Huancavelica }\end{array}$ & $1 \%$ & $1 \%$ & $0 \%$ & $0 \%$ & $0 \%$ & $0 \%$ & $0 \%$ & $0 \%$ & $0 \%$ & $0 \%$ & $0 \%$ & $0 \%$ & $0 \%$ \\
\hline Ferrocarril Tacna - Arica & $0 \%$ & $0 \%$ & $0 \%$ & $0 \%$ & $0 \%$ & $0 \%$ & $0 \%$ & $0 \%$ & $0 \%$ & $0 \%$ & $0 \%$ & $0 \%$ & $0 \%$ \\
\hline
\end{tabular}

Fuente: Empresas Ferroviarias. Elaboración: MTC-OGPP-Oficina de Estadística: 2004-2016.

El parque ferroviario ha tenido una tasa de crecimiento negativo de $11.45 \%$ en términos globales. Para el año 2004 se contaba con 2.981 unidades vehiculares, ésta cifra para el año 2016, sufriría una reducción de 341 unidades, llegando a 2.640 unidades vehiculares. Desglosando con respecto al tipo de vehículo, las locomotoras, autovagones, autocarriles y coche de pasajeros, tuvieron un dinamismo positivo; mientras que el tipo de vehículo vagón de carga, tuvo un dinamismo negativo, para el año 2004 se contaba con 2.719 unidades, sufriendo un descenso de 404 unidades para el año 2016 , con un total de 2.315 unidades. Tal como se ve en la tabla 5.

Tabla 5. Parque ferroviario, según tipo de vehículo: 2004-2016 (Número de vehículos).

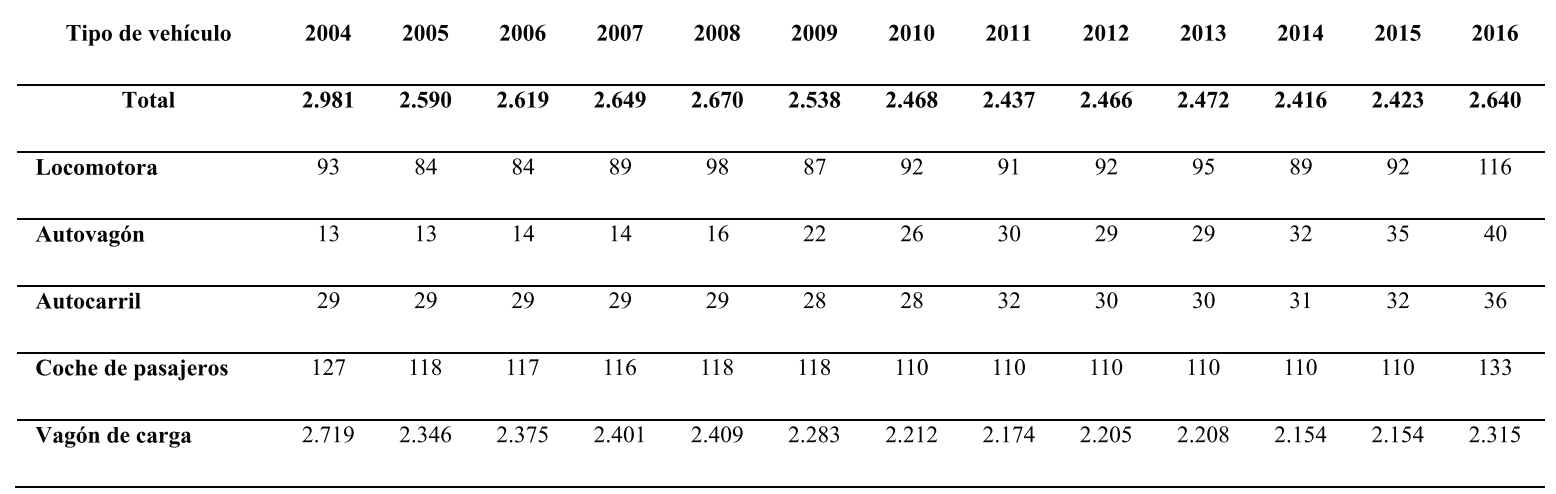

Fuente: Empresas Ferroviarias. Elaboración: MTC-OGPP- Oficina de Estadística: 2004-2016.

\section{DISCUSIÓN}

Primero: el transporte ferroviario peruano es un sistema no integrado con los demás modos de transporte. La característica de un país para que sea competitivo, es contar con infraestructura instalada de red de transporte de carga y pasajeros y que esto sea integrado a los demás modos de transporte, lo cual como efectos externos genere economías de escala y con ello los costos de transporte se reducen de manera significativa.

La infraestructura en el sistema de transporte ferroviario no ha tenido mucha expansión con respecto al kilometraje, y tampoco se ha priorizado un plan de interconexión entre ellas mismas ni con los demás modos de 
transporte. Para el año 2010, el Perú contaba con $1.906,6 \mathrm{~km}$ de extensión del sistema de transporte ferroviario, y para el año 2016, solo se ha incrementado en $33,1 \mathrm{~km}$. Esto representa la inversión hecha en el Metro de la Línea 1.

El Ferrocarril del Centro y del Sur, y otros que existen, no están interconectados. Cada uno de ellos opera de forma separada. El Ferrocarril del Centro tiene mayor incidencia en el traslado de carga, y el Ferrocarril del Sur y Sur Oriente, una mayor incidencia en el transporte de pasajeros.

Segundo: la evolución del mercado del transporte ferroviario ha sido limitada y la inversión en los 2004 y 2016, no fue significativa. Solo se ha hecho reinversiones para poder mantener la infraestructura operativa. Las concesiones hechas en el Ferrocarril de Centro y del Sur y Sur Oriente, no contempla inversiones obligatorias de expansión, ni proyectos nuevos.

En consecuencia la infraestructura ferroviaria no ha sufrido cambios considerables en el periodo de análisis. El parque ferroviario, en los años analizados ha sufrido una disminución del $11.45 \%$, es una variación relativa considerable negativa.

La concesión busca lograr la eficiencia económica, y con ello el bienestar económico. Tanto en el Ferrocarril del Centro y del Sur y Sur Oriente, la desintegración fue vertical, y con ello se busca la entrada de nuevas empresas en la prestación de servicio de carga y pasajero, lo cual no ha tenido los efectos esperados, ni un incremento de un mayor dinamismo en el sector. La entrada de nuevos competidores, no ha sido efectiva, si no es efectiva existe mucha limitación al acceso a una economía de mercado competitivo.

\section{CONCLUSIONES}

La competitividad de un país está estrechamente relacionada con el nivel de inversión y desarrollo de la infraestructura en la red de transporte para el traslado de carga y pasajeros. El desarrollo de la infraestructura en el transporte ferroviario ha sido limitado, y tampoco tuvo un impacto creciente, y una integración a los otros modos de transporte en el territorio peruano. La interconexión y la expansión del Ferrocarril del Centro y del Sur y Sur Oriente han sido escasas.

El sistema de transporte ferroviario en los años analizados no ha mostrado un dinamismo, como se esperaba, en la expansión del servicio ni en la competencia del sector. Las concesiones hechas fueron por periodos muy prolongados, lo cual frena la innovación y la apertura del mercado.

La inversión en el mercado del transporte ferroviario no ha tenido una tasa de crecimiento positiva, más bien se tuvo una contracción en el parque ferroviario; lo cual podría llevar a concluir, que esta contracción obedezca, a una regulación inadecuada del sector y una estructuración deficiente de las concesiones realizadas.

\section{REFERENCIAS BIBLIOGRÁFICAS}

Banco Mundial. (2011). La reforma de los ferrocarriles: Manual para mejorar el rendimiento del sector ferroviario. Recuperadodehttps://ppiaf.org/sites/ppiaf.o $\mathrm{rg} /$ files/documents/toolkits/railways_toolki t/index_sp.html

Comisión Económica para América Latina y el Caribe. (2012). Perfiles de infraestructura y transporte en América Latina. Caso Perú. Unidad de Servicios de Infraestructura. 141.

Corporación Andina de Fomento (2004). Perú: Análisis del sector transportes. $\mathrm{N}^{\mathrm{o}} 3$. Recuperadodehttp://www.scioteca.caf.com /handle/123456789/395

Donges, J. (1991). Deregulating the German Economy. Occasional papers. Number 15. $1-40$.

Gwilliam, K. (2001). Competition in Urban Passenger Transport in the Developing World. Journal of Transport Economics and Policy. Volume 35. Part 1, 99-118.

Pastor, C. \& Pérez, P. (2009). El reto de la infraestructura al 2018: La brecha de inversión en infraestructura en el Perú 2008. Recuperado de http://ipe.org.pe/wpcontent/uploads/2009/09/estudio_el_reto_d e_la_infraestructura_al_2018.pdf

Ruiz, F. \& Ludeña, C. (2008). Sobrecostes de transporte: ¿Maldición geográfica o falla de politica de infraestructura? Una estimación para Paraguay desde un modelo de equilibrio general. Cuaderno de economía. $\mathrm{N}^{\mathrm{o}} 3,59-82$. 
Ruiz-Rua, E. (2010). Liberalización, efectos en los servicios ferroviarios: el caso del servicio de alta velocidad Madrid-Barcelona. Revista Económica, Rio de Janeiro. V12, N2. 15-42.

Rufián, D. (2002). Políticas de concesión vial: Análisis de las experiencias de Chile, Colombia y Perú. CEPAL- Serie Gestión Pública. $\mathrm{N}^{\circ}$ 16. 1-76.

Thomson, I. (1998). La evolución del papel del Estado en la regulación del transporte terrestre. Revista de la CEPAL. N 64, 149163.

Thomson, I. (1999). Concessions and optimization: road and rail transport. Cepal Review, $\mathrm{N}^{\mathrm{o}}$ 67. 177-189.

Thomson, I. (2001). El desarrollo institucional del transporte en América Latina durante los últimos veinticinco años del siglo veinte. Serie de recursos naturales e infraestructura. $\mathrm{N}^{\mathrm{o}} 17.1-42$. 